\title{
Analysis of the Effect of Virtual Reality Technology on Improving Drug Design
}

\author{
Xinqiao Luo
}

School City, University of California, Santa Cruz, 95129, United States

shilishuang@cas-horbour.org

\begin{abstract}
Virtual reality technology is a technology that has gained popularity in recent days in the wake of the fourth industrial revolution. It has been widely studied and used across different medical applications. In the pharmaceutical industry, virtual reality can be applied as a replacement or adjunctive for pharmacotherapy. Virtual reality in improving drug design has presented several benefits, although it is yet fully implemented. For instance, VR tools have been used in pharmacist education, drug design, discovery, behavior modification, and patient counseling. The interest in researching virtual reality is continuously increasing despite several limitations, such as financial constraints. This research makes conclusions through analyzing secondary sources. Amidst this, past research on the topic has proved that virtual reality technology is completely safe and much more effective, an aspect that can promote user satisfaction. Furthermore, it is slowly becoming affordable, portable, and even flexible, an aspect that has smoothened therapeutic processes for outpatient and inpatient environs. Some of the other challenges identified by this research include usability issues such as costs, validation of its efficacy, and acceptance.
\end{abstract}

Keywords: Virtual reality, Drug design, Pharmaceutical industry, Virtual reality simulation

\section{INTRODUCTION}

Technology today has transformed the whole production unit into a new level. Virtual reality (VR) technology has progressively been used by different researchers in a wide range of fields over the recent years. It has been consequently integrated into different aspects of human life, such as video games to other different industrial sectors. For instance, the Covid-19 pandemic is one pandemic that hugely integrated virtual reality in developing drugs to counter the disease and reduce its effects. In the wake of these developments, virtual reality is starting to be integrated into rational drug design and drug development and discovery. This paper aims to discuss the application of virtual reality in drug development and some benefits it brings to the pharmaceutical industry.

The research shall be significant to understand virtual reality technology and some of the different ways it can be integrated into drug design. The research shall also form a basis for future studies on the topic where other developments can also be made based on the research. Virtual reality has been integrated into several functions across the drug industry. The article contest that virtual reality technology can enhance people's understanding of the disease progression and enable the provision of means of designing and testing new treatments for the diseases. Also, the research seeks to identify the different VR technologies used in improving drug design and also showcase the different outcomes of incorporating VR in drug design. E-Brain is one virtual reality platform used to enable drug administration to the brain and assists drug uptake and diffusion.

\section{ANALYSIS}

\subsection{Application of Virtual Reality Technology in Medical Field}

Virtual reality technology in healthcare and medical research has been used to enhance and improve user experience and performance in correcting segmentation errors. The technology has gained more attention in different medical fields such as training, imaging, and diagnostic tools, an aspect that has enabled drug design to develop to another new level. For instance, VR-based software, which is progressively to guide users, has enabled the reduction of MRI segmentation errors. The virtual reality technology-aided software has been used 
to provide a more intuitive, efficient, and engaging alternative to the formerly used techniques[1] For example, the technology has been utilized in surgical training to provide VR-based simulations to develop stem education. different errors have been minimized without VR technology in the drug industry as we edge closer to accuracy[2]. Virtual reality technology has been relevant and successfully utilized to release stress and pain reduction $\mathrm{n}$ for patients with different critical chronic ailments. Virtual reality technology is consistently used to monitor, diagnose, and aid patients through the virtual world, hence easier addressing their conditions.

Virtual reality technology can supplementary be used in accelerating the development of drugs by providing an innovative technology-based framework where ideas can be developed in greatly engineering drug products. For instance, the development of virtual reality simulations using experimental knowledge can enhance the reverse engineering process[2] This is one of the biological systems that are vital in the creation of insights into the function. Biologically driven mechanisms can explain different explanations, hence providing answers to the different questions in the research[3]. The virtual reality simulation research process provides a bridge in delving deeper into different areas that are not yet understood fully. An understanding of the system level of a particular system can be modeled under the development of VR technology. The process focuses on the different interactions in the parallel and interacting mechanisms, enabling the researchers to identify new targets in the development of drugs. Additionally, the physicochemical properties of drugs are tested under the simulations. Thus interactions of the drugs with their target trails can be predicted. Drug repurposing analysis can be done under the wake of VR technology, an aspect that could not be done much easier before.

\subsection{Advantages of using virtual reality technology}

There are several benefits aligned to the use of VR technology in the development of drugs. First, several drugs are composed of small molecules which can be better analyzed using the latest technology. For instance, virtual reality can be used in developing drugs that can bind target cells. The structure-based drugs are now easily produced at the eyes VR tools[4]. Second, based on research, drugs must be tested and proven safe and efficient before they are used on actual patients. With virtual reality, this function has been simplified, and accuracy increased. Third, the VR tools have played a significant role in the designing and testing the manufactured drug products. This aspect has aided in reducing complications that arise out of the usage of inefficient and untested drugs that can be deemed unfit for human consumption[3]. Finally, the tool of virtual reality is one significant aspect that cannot be ignored in the achievement of research goals across the different fields it has been used. Drug development is now achieving perfection as further developments are made in drug research and development.

The pharmacodynamics of drugs has been novellayered by virtual reality simulations, which have aided in analyzing drug effects under simulated theoretical systems in a 3-D environment. Data sets from different subjects can be used to construct simulations which can enable the simulations to imitate precise conditions that can be used to analyze different scenarios for different patients. Virtual reality technology is essential in providing means for pharmacokinetic studies by testing the process through absorbing, distributing, metabolism, and excreting the drugs through diverse delivery systems[5] Virtual reality thus provides a different alternative to carry out the pharmacodynamics/pharmacokinetic models in guiding the forecasts on the most efficient treatment strategies, drug efficacy, and even drug concentration[6]. This aspect helps improve drug testing and is also a process that accelerates the process of new drug screening and is essential in the provision of data essential to the Investigational New Drug apps. The virtual reality simulations are thus used to provide suggestions for changes and predict new possible indications to administration of drugs, hence determining the optimal dosage levels.

Moreover, virtual reality technology is useful in aiding patient populations stratifications, especially in clinical trials centered on data classifications, genetic mutations, symptoms, and other specific criteria. The virtual reality technology simulations are also significant in aiding the shortening of lead generation and screening points in pre-clinical works through theoretical testing. They can be used to make necessary efficacy predictions[5]. Virtual reality has been used to develop multimodal and interactive sensory stimuli and has presented unique benefits over other computer-based techniques in molecular-level applications and other scientific researches. Also, virtual reality simulations are vital because they can be developed based on specific patient information, which can be used to predict and make the best treatments for the specific patients by utilizing the simulations' predictions[6]. Practically, although patients have similar ailments, sometimes practitioners have different prescriptions for the patients. These prescriptions can be made more accurate and specific to the patients with virtual reality technology.

However, besides the huge benefits, it is a complicated and expensive technology to be adopted by several users that need it. Analysis of drug side effects has been made easier in developing virtual reality simulations in drug development and designing. It has enabled researchers to grab hold of virtual molecules. Hence they can note their movements and respond to different 
stimuli[5]. We all see these benefits in the books, but nothing is benefiting if it is not helping you. Besides, VR technology in drugs requires sufficient research to enable its efficacy. Without sufficient research in VR, its adoption in healthcare can be hindered. This can make people conclude virtual reality as 'virtual reality[6]. This is because it exists, but it is quite costly to incorporate and use by people who need it. Besides, most of the end-users are yet to know the benefits of VR. Lastly, virtual reality in drug design requires a virtual pharmacy, an aspect that is very hard to achieve practically.

\section{DISCUSSION}

To begin, virtual reality is yet to incorporate artificial intelligence in the processes of drug discovery and development. The use of artificial intelligence in drug production and development will further the efficacy of the whole process (Yuan, Chan, and $\mathrm{Hu}, 2017$ ). Many start-ups in the medical field are venturing into the incorporation of machine technologies that are compatible with virtual reality to counter the different challenges that have been consistent in the pharmaceutical industry. For instance, patient identification is one of the most important parts of drug discovery and development[7]. With the aid of artificial intelligence and virtual reality, the step shall be greatly simplified, and the costs incurred shall also be reduced sequentially. Virtual reality is used in drug designing and development; artificial intelligence will therefore be used to complete the role started b virtual reality. Some companies have used artificial intelligence to identify the best drugs for certain diseases[7]. Thus, aligning virtual reality technology with artificial intelligence, the industry's best results shall be used.

The future will witness a complete level of new drug strategies. Traditional medicines will no longer be produced and sold out with no follow-up or technological intrusion. Digital health technology shall be greatly used in the future, and fewer errors shall be encountered. For instance, people in the future shall be able to detect their health using simple gadgets[8]. For example, patients with diabetes shall monitor their sugar levels by simply using their smartphones without necessarily possessing medical equipment. The technology shall also allow the patients to possess enjoyable experiences with their healthcare sector[9]. Virtual reality holds a bright future for patients. With drug prescriptions being made virtually without the physical participation of the patients, drug side effects can be easily detected, and they can thus be easily combated.

Lastly, with the various inventions in virtual reality, mixed reality, and augmented reality, visualizations in the future shall be made like never before. More pharmaceuticals shall consider going for the hidden possibilities of these technologies in their drug research, design, and development. Imagine when the pharmaceutical industries will no longer require live animals to test their drugs on before they manufacture. There shall no longer be guinea pigs for drug tests because all it requires is to conduct the drugs on computer simulations. For instance, during the Covid-19 pandemic, the vaccine to the drug was tested on a few individuals who offered themselves. This could be dangerous because nobody knew the side effects of the drugs and the perfect dosage for the individuals[10]. With the use of artificial intelligence, the manufacture and testing of such drugs shall be changed completely.

\section{CONCLUSION}

Although virtual reality is yet to be completely incorporated in drug design and development, it still has a vital potentiality. If researchers commit themselves and delve deeper into the topic, more information shall be acquired and shall be made available to improve efficacy within the field. Although the current production costs and other costs are still high, with more developments in the field, the costs shall be significantly reduced and reduce the number of obstacles. This shall further improve the sage of virtual reality and make it a viable and important clinical instrument in the medical research industry. Virtual reality has changed the whole perspective of drug development and testing, placing the exercise on a different level. The technology has gained more attention in different medical fields such as training, imaging, and diagnostic tools, which has enabled drug design to develop to another new level. Virtual reality technology is consistently used to monitor, diagnose, and aid patients through the virtual world, hence easier addressing their conditions.

As the technology enhances drug production and usage efficacy, the complete process in the healthcare field shall be completely changed as the knowledge shall be regarded as an important tool in medical education, research, and patient counseling. The virtual reality simulation research process provides a bridge in delving deeper into different areas that are not yet understood fully. An understanding of the system level of a particular system can be modeled under the development of VR technology. The VR tools have played a significant role in the designing and testing of the manufactured drug products. This aspect has aided the reduction of complications that arise out of usage of inefficient and untested drugs that can be deemed unfit for human consumption. Electronic patient data records will enable the virtual-reality tools to collect patient data and utilize it in the drug designing and development sector.

\section{ACKNOWLEDGMENT}

This is to acknowledge all those whose help was a significant factor in the success of my project. Under a very short timeframe, I successfully completed the research. It could not have come true without the help of 
my dear parents and friends, who offered me moral support and other forms of guidance throughout the project. Also, I would love to submit my special thanks to the lecturer (NAME) who presented this precious opportunity to tackle the project. Without the project, I would not know much more about what I discovered within the subject. I have learned a lot of new things, and I am now able to see things differently.

\section{REFERENCES}

[1]Hernández-Rodríguez, Maricarmen, Martha C Rosales-Hernández, Jessica E Mendieta-Wejebe, Marlet Martínez-Archundia, and Jose Correa Basurto. "Current tools and methods in molecular dynamics (MD) simulations for drug design." Current medicinal chemistry 23, no. 34 (2016): 3909-3924.

[2]Dalkas, Georgios A., Dimitrios Vlachakis, Dimosthenis Tsagkrasoulis, Anastasia Kastania, and Sophia Kossida. "State-of-the-art technology in modern computer-aided drug design." Briefings in bioinformatics 14, no. 6 (2013): 745-752.

[3]Brown, Nathan, Peter Ertl, Richard Lewis, Torsten Luksch, Daniel Reker, and Nadine Schneider. "Artificial intelligence in chemistry and drug design." (2020): 709-715.

[4]Coyne, Leanne, Thayer A. Merritt, Brittany L. Parmentier, Rachel A. Sharpton, and Jody K. Takemoto. "The past, present, and future of virtual reality in pharmacy education." American journal of pharmaceutical education 83, no. 3 (2019).

[5]Noori, Hamid R., and Rainer Spanagel. "In silico pharmacology: drug design and discovery's gate to the future." (2013): 1-2.

[6]Khan, Tahmeena, Rumana Ahmad, Iqbal Azad, Saman Raza, Seema Joshi, and Abdul Rahman Khan. "Computer-aided drug design and virtual screening of targeted combinatorial libraries of mixed-ligand transition metal complexes of 2butanone thiosemicarbazone." Computational biology and chemistry 75 (2018): 178-195.

[7]Schneider, Petra, W. Patrick Walters, Alleyn T. Plowright, Norman Sieroka, Jennifer Listgarten, Robert A. Goodnow, Jasmin Fisher et al. "Rethinking drug design in the artificial intelligence era." Nature Reviews Drug Discovery 19, no. 5 (2020): 353-364.

[8]Verma, Saroj, and Yenamandra S Prabhakar. "Target based drug design-a reality in virtual sphere." Current medicinal chemistry 22, no. 13 (2015): 1603-1630.
[9]Xu, Kui, Nan Liu, Jingle Xu, Chunlong Guo, Lingyun Zhao, Hong-Wei Wang, and Qiangfeng Cliff Zhang. "VRmol: an integrative cloud-based virtual reality system to explore macromolecular structure." BioRxiv (2019): 589366.

[10]Yuan, Shuguang, HC Stephen Chan, and Zhenquan Hu. "Implementing WebGL and HTML5 in macromolecular visualization and modern computer-aided drug design." Trends in biotechnology 35, no. 6 (2017): 559-571. 